\title{
miR-506 suppresses cervical cancer cell proliferation both in vitro and in vivo
}

\author{
M. GONG, C. CHEN ${ }^{*}$, H. ZHAO, M. SUN, M. SONG \\ Department of Obstetrics and Gynecology, Capital Medical University Affiliated Beijing Chaoyang Hospital, 100043, Beijing, P. R. China \\ ${ }^{*}$ Correspondence: vqc819@163.com
}

Received January 12, 2017/ Accepted July 12, 2017

\begin{abstract}
Cervical cancer (CC) is one of the most common gynecological malignancies in women worldwide. Recently, increasing evidence indicates aberrant expression of miR-506, which was reported to be associated with a variety of tumors. The aim of this study was to evaluate the potential role of miR-506 in CC and to verify its effect on the regulation of ABCC4. The expression of miR-506 in cervical cancer tissues, HeLa and C33A cell lines was examined using quantitative Real-time PCR. MTT assay and animals studies were used to examine the effects of miR-506 on cervical cancer proliferation. Luciferase reporter and western blot were used to confirm that miR-506 could regulate ABCC4. We found that miR-506 was significantly downregulated in human CC cell lines (HeLa and C33A) and clinical CC specimens as compared to matched cell lines and adjacent normal tissues, while the expression level of ABCC4 was higher in tumor tissues than in the adjacent normal tissues. We also revealed that up-regulated expression of miR-506 could inhibit CC cells proliferation both in vitro and in vivo. Moreover, ABCC4 was identified as a direct target of miR-506 and the inverse relationship between them was also observed. In summary, our findings suggest that miR-506 has an important role in suppressing CC cell proliferation and suppresses the expression of ABCC4 by directly targeting its 3'-UTR. miR-506 may represent a novel therapeutic target of microRNA-mediated suppression of cell proliferation in CC, but the role of the miR-506/ABCC4 axis in CC progression needs further study.
\end{abstract}

Key words: miR-506, ABCC4, cervical cancer, proliferation

Cervical cancer (CC) is the second most frequent gynecological malignancy in women around the world [1], and almost $90 \%$ of CC-related deaths occur in developing scale of the world [2]. Human papillomavirus (HPV) infection is one of the most important risk factors in CC development and is detected in about $99 \%$ of cervical tumors [3]. However, some studies have revealed that HPV infection is not sufficient to develop CC alone [4], other risk factors, such as heterosexual women, women with multiple sexual partners, lower social class, immunosuppression, smoking, and non-attendance screening program, should be pivotal in the progression of CC simultaneously. Although many advanced protocols including operation, chemotherapy and radiotherapy have been made in the treatment of CC, the long-term outcomes of clinical therapy remain unsatisfactory [5]. Nevertheless, with the intensive studies on the molecular mechanism of progression and carcinogenesis of CC that began to accumulate, various new molecules which are likely to be the targets of CC were identified [6]. Therefore, it is urgent to find the underlying molecular mechanisms of CC and identify the promising therapeutic targets to improve treatment strategies.
MicroRNAs (miRNAs) are a subset of small, highly conserved, and non-coding RNAs with a size of 21-24 nucleotides that bind to the 3 '-untranslated region (3'-UTR) of target messenger RNAs (mRNAs) to induce translational degradation or repression [7]. Increasing evidence has manifested that miRNAs play a critical role in regulating cancer progression and are involved in cancer cell death, differentiation, proliferation, metastasis, and apoptosis [8-10]. In the recent decades, many studies have shown that the expression of various miRNAs was found to be dysregulated in CC [11-13]. MiR-506 was identified as a member of the miR-506-514 cluster, which includes seven distinct miRNAs: miR-506, -507, -508, -509, -510, -513 and miR-514 $[14,15]$. Besides, its abnormal expression was detected to be associated with multiple benign diseases and malignant carcinomas [16-20]. Wen et al reported that miR-506 exerted its anti-proliferative function by directly targeting Gli3 in human CC [21]. However, the specific functional molecular mechanisms of miR-506 in CC are still elusive, and the potential of miR-506 as a therapeutic target of CC remains to be evaluated. 
ATP-binding cassette sub-family C member 4 (ABCC4), also named multidrug resistance protein 4 (MRP4), belongs to the ATP binding cassette family of membrane transporters subfamily $\mathrm{C}$ (ABCC) and mediates ATP-dependent transport of various organic anions, including sulfated steroids, cyclic nucleotides, reduced folates and glucuronidated $[22,23]$. Based on its high variability in function and expression, ABCC4 relates to the onset and prognosis of several malignant diseases, and Deanna L. Kroetz et al has reported that ABCC4 was directly regulated by miR-506 in human kidney [24].

In this study, we demonstrate that miR-506 is frequently downregulated and significantly correlates with aggressive clinicopathological characteristics in CC. The re-expression of miR-506 in CC cells remarkably suppressed cell proliferation in vitro and in vivo. Furthermore, we identified ABCC4 as a direct and functional target of miR-506. The newly identified miR-506/ABCC4 axis partially elucidates the molecular mechanism of CC proliferation and represents a novel potential therapeutic target for CC treatment.

\section{Materials and methods}

Tissue specimens. Fifty paired cases of the CC and the normal squamous epithelial tissues were collected at Department of Obstetrics and Gynecology, Beijing Chaoyang Hospital (Beijing, China), from 2014 to 2016. The average age of the fifty patients was 40 years (range 18-69). All tissues obtained from biopsy or surgery were frozen immediately in liquid nitrogen and stored at $-80^{\circ} \mathrm{C}$ until RNA extraction. Neither radiotherapy nor chemotherapy therapy was used in any patients before surgery. Histopathological diagnoses were based on the WHO classification and clinical stages classification was following the International Federation of Gynecology and Obstetrics criteria (FIGO). The characteristics of patients with CC are described in Table 1. Informed consent was obtained from all patients. Collection and experimentation of human tissues were approved by the Ethics Committee of Beijing Chaoyang Hospital in accordance with the Declaration of Helsinki.

Cell culture. Primary normal cervical squamous cells (NCSC) and two human CC cell lines (HeLa and C33A) obtained from Shanghai Institute of Cell Biology (Shanghai, China) were maintained in RPMI 1640 medium (Gibco, Carlsbad, CA, USA) supplemented with $10 \%$ fetal bovine serum (FBS, Sigma) at $37^{\circ} \mathrm{C}$ in an atmosphere consisting of $5 \% \mathrm{CO}_{2}$.

RNA isolation and quantitative real-time PCR (qRT-PCR). Total RNA was isolated and purified using a RNA simple Total RNA Extraction Kit (TIANGEN, Beijing, China) according to the manufacturer's instructions. To perform the quantitative detection of miR-506 or mRNA expression, first strand cDNA was reverse-transcribed and then qRT-PCR was carried out using a SYBR Prime Script miRNA RT-PCR kit (Takara, Otsu, Japan) according to the manufacturer's protocol. Glyceraldehyde-3-phosphate or dehydrogenase (GAPDH) or U6 small nuclear RNA (HAPK Biotechnology, Shenzhen, China) was used as an internal control. The $2^{-\Delta \Delta \mathrm{Ct}}$ method was applied to quantify the relative expression levels of miRNA or mRNA. All the qRT-PCRs were run in triplicate. All primers were designed and synthesized by RiboBio (Guangzhou, China). The primers used were as followed: miR-506 forward, 5'-TAAGGCACCCTTCTGAGTAGA-3', reverse, and 5'-GCGAGCACAGAATTAATACGAC-3'; U6 forward, 5'-AGAGCCTGTGGTGTCCG-3', and reverse, 5'-CATCTTCAAAGCACTTCCCT-3'. ABCC4 forward 5'-GGCAGTGACGCTGTATGG-3', and reverse, 5'-CGCCAGGTCTGACAGTAAAG-3'. GAPDH forward, 5'-GACTCATGACCACAGTCCATGC-3', and reverse, 5'-AGAGGCAGGGATGATGTTCTG-3'. The synthesis of first cDNA and qRT-PCR were performed as previously described [25]. Briefly, the reference sequence for Homo sapiens miR-506 (NR_030233.1) was obtained from Pubmed Genbank. The sequences of forward and reverse primers were analyzed in OligoCalc web-based software to check for self-complementarity and hairpin formation of primers. Optimized PCR conditions were obtained by performing reactions in different concentrations of $\mathrm{MgCl}_{2}$ and a spectrum of annealing temperatures. PCR cycling conditions were as follows: an initial denaturation step at $95^{\circ} \mathrm{C}$ for $10 \mathrm{~min}$, followed by 40 cycles of denaturing at $95^{\circ} \mathrm{C}$ for 10 $\mathrm{sec}$, annealing and synthesis at $60^{\circ} \mathrm{C}$ for $60 \mathrm{sec}$. The relative expression levels were calculated by comparing $\mathrm{Cq}$ values of the samples with those of the reference $\left(2^{-\Delta \Delta \mathrm{Cq}}\right)$, and all data were normalized to the internal control GAPDH [26].

Plasmid constructs. The miR-506 mimics, miR-506 inhibitor, and miR-506 negative-control were synthesized and purified by RiboBio (Guangzhou, Guangdong, China); siABCC4 and anti-siABCC4 were purchased from RiboBio, as illustrated in Table 2. For the construction of the lentivirus vector expressing miR-506, the oligonucleotides were cloned into the pGreenPuro vector (System Biosciences, Mountain View, CA, USA) and the empty vector was used as the control [27]. The pGreenPuro vector containing two restriction enzymes cutting sites was linearized by restriction digest with BamHI and EcoRI. Then, the oligonucleotides were cloned into the linearized vector, and the identification of clones was performed subsequently. For screening of miR-506 insertion, the H1 PCR primers were designed: forward, 5'-AATGTCTTTGGATTTGGGAATCTTAT-3'; reverse, 5'-TGGTCTAACCAGAGAGACCCAGTA-3'. All the steps of plasmid construction were based on the user manual and previous studies $[28,29]$. The lentiviruses were produced, and their titers were determined by Lenti-Pac ${ }^{\mathrm{TN}} \mathrm{HIV}$ kit (GeneCopoeia). Then, cultured cells were transduced according to the previously described methods [30]. To construct the ABCC4 expression plasmids, the full-length construct with wild-type 3'-UTR or mutant 3'-UTR of ABCC4 gene was cloned into the PEZ-Lv105 lentivirus vector (GeneCopoeia). The ABCC4 3'-UTR sequence was amplified from human cDNAs by 
PCR. The wildtype (WT) and mutated (MT) 3'-UTR regions of ABCC4 were cloned into Dual-Luciferase miRNA Target Expression Vector (Promega, Madison, WI, USA). Point mutations in the putative miR-506 binding seed regions were created using the Quick-Change Site-Directed Mutagenesis kit (Stratagene) according to the manufacturer's protocol. All constructs were verified by sequencing.

Transfection. Stable transfections were performed using Lipofectamine 2000 (Invitrogen) according to the recommended instructions and previous methods described by other studies [29]. Because the infected cells stably express copGFP and puromycin, they can be selected either for GFP-positive cells by FACS of for puromycin resistance by puromycin treatment. In this study, we generated a killing curve for the target cells with different concentrations of puromycin in a 96-well plate. Stable cells were selected in the presence of puromycin (at the final concentration of $5 \mu \mathrm{g} / \mathrm{ml}$ ) for 5-7 days with repeated plating. All of the siRNAs and miRNA mimics were synthesized by RiboBio (Guangzhou, Guangdong, China). They were transiently transfected with siRNA mate (RiboBio). The siRNAs were used at a concentration of $20 \mu \mathrm{m}$, miRNA mimics were used at concentration of $20 \mathrm{~nm}$, and the sequences are shown in Table 2.

Luciferase reporter assay. The ABCC4 3'-UTR sequence was amplified from human cDNAs by PCR. The wildtype (WT) and mutated (MT) 3'-UTR regions of ABCC4 were cloned into Dual-Luciferase miRNA Target Expression Vector (Promega, Madison, WI, USA). The miR-506 mimics, miR-506 inhibitor and negative control were purchased from RiboBio (Guangzhou, Guangdong, China). The reporter plasmids were co-transfected with miR-506 mimics/inhibitor or the control into cervical cancer cells using Lipofectamine 2000 (Invitrogen) in 24-well plates. Luciferase assay was performed using the Dual-Luciferase ${ }^{\circledast}$ Reporter Assay System (Promega) two days after transfection.

Cell proliferation assay in vitro. Cell proliferative activity was determined using the 3-(4, 5-dimethyl-2-thiazolyl)-2, 5-diphenyl-2-H-tetrazolium bromide (MTT) method (Beyotime Shanghai, China). HeLa and C33A cells, transiently transfected with the miR-506 mimic, miR-506 inhibitor, negative control for $24 \mathrm{~h}$, were seeded in 96-well plates at a density of $5 \times 10^{3}$ cells per well in $100 \mu \mathrm{L}$ of growth medium and cultured for another $24 \mathrm{~h}, 48 \mathrm{~h}$ or $72 \mathrm{~h}$, respectively. Subsequently, $10 \mu \mathrm{L}$ of MTT solution $(5 \mathrm{mg} / \mathrm{mL})$ was added to each well. After $4 \mathrm{~h}$ incubation at $37^{\circ} \mathrm{C}$, the cells were disrupted in $200 \mu \mathrm{L}$ of dimethyl sulfoxide (DMSO, Sigma-Aldrich), and the absorbance was measured at 490 $\mathrm{nm}$ on a microplate reader (BioTeke, Winooski, VT, USA).

In vivo nude mice tumorigenesis and proliferation experiments. BALB/c nu/nu female mice at 4 weeks of age were provided and housed in the Laboratory Animal Center of Shanghai Institutes for Biological Sciences. All the experiments with live animals were approved by the Ethics Committee of Beijing Chaoyang Hospital and were conducted in compliance with the Guide for Care of
Laboratory Animals as detailed by the National Ministry of Science. As previously described, the cervical cancer model in BALC/c mice was established. After 4 days of acclimatization, $2 \times 10^{5}$ HeLa cells stably infected with either miR-506 mimics or miR-control were injected subcutaneously into the dorsal flanks of each mice (ten in each group). The tumor size was measured every 4 days. Then the mice were killed and necropsies were performed on the $28^{\text {th }}$ day after injection. Then, the tumor volumes were calculated following the formula: $\mathrm{A} \times \mathrm{B} 2 / 2$ ( $\mathrm{A}$ : the largest diameter, $\mathrm{B}$ : the diameter perpendicular to $\mathrm{A})$.

Protein extraction and western blotting. All proteins were extracted from cells with radio immune precipitation assay (RIPA) lysis buffer (Solarbio, Beijing, China), supplemented with a protease inhibitor, and separated by $10 \%$ SDS-PAGE. The separated proteins were transferred to the polyvinylidene fluoride (PVDF, Millipore, Boston, MA, USA) membrane. The membrane was blocked with 5\% nonfat milk and incubated with a specific primary antibody at $4{ }^{\circ} \mathrm{C}$ overnight. A horseradish peroxidase-conjugated secondary antibody (Santa Cruz, CA, USA) was incubated for $1 \mathrm{~h}$ at $25^{\circ} \mathrm{C}$. $\beta$-actin primary antibody (1:5000 dilution; ZSGB-BIO, Beijing, China) was chosen as the internal reference. The proteins of interest were revealed by the Electro chemiluminescence (ECL) detection reagents (Sea Biotech, Shanghai, China). Lab Works ${ }^{\mathrm{TM}}$ Image acquisition and analysis software (UVP, Upland, CA, USA) was used to quantify the intensities of the band.

Statistical analysis. Statistical analyses were carried using the SPSS 17.0 program (SPSS, Chicago, IL, USA). Data were presented as the mean \pm SD from at least three independent experiments. The statistical significance between groups was determined using the Student's t-test. The relationship between clinicopathological characteristics and miR-506 expression was analyzed using Pearson $\chi^{2}$ test. P-value less than 0.05 was considered to be statistically significant.

\section{Results}

miR-506 expression is downregulated and inversely correlated with ABCC4 expression in cervical cancer. The expression levels of miR-506 were detected in 50 matched pairs of women $\mathrm{CC}$ tissues and adjacent normal tissues by qRT-PCR. In comparison to the non-tumor cervical tissues, miR-506 expression was dramatically lower in the CC tissues (Figure $1 \mathrm{~A}, \mathrm{p}<0.001$ ). In addition to the CC tissues samples, we further detected the relative expression levels of miR-506 in two cell lines (HeLa and C33A) and compared them with the normal cells. The results showed the miR-506 expression levels in HeLa and C33A cell lines were significantly reduced relative to that in the normal cell NCSC (Figure 1B, $\mathrm{p}<0.05$ ).

Furthermore, the expression levels of ABCC4 in the CC tissues and the adjacent normal tissues were also assessed by $\mathrm{qRT}$-PCR. As shown in Figure 1C, the relative expression level of ABCC4 was significantly higher in the CC tissues 
A

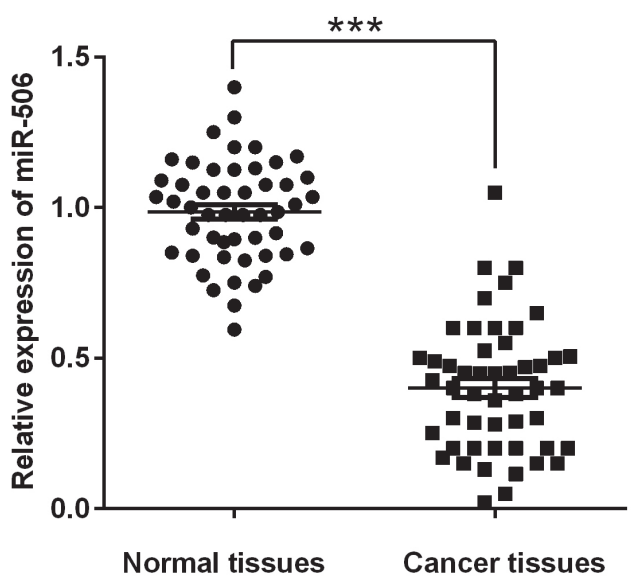

C

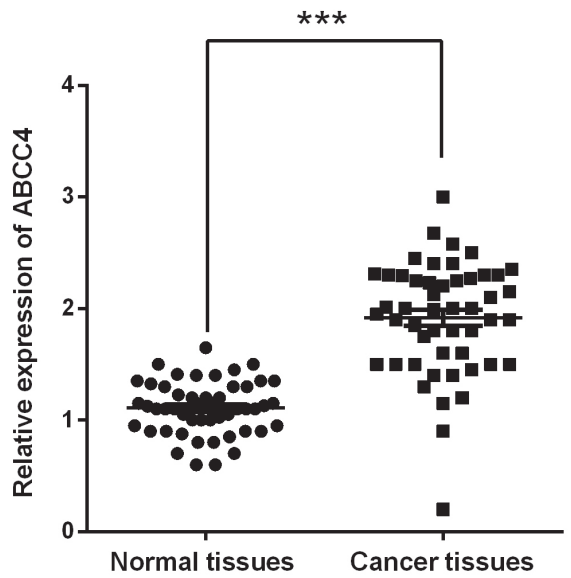

B

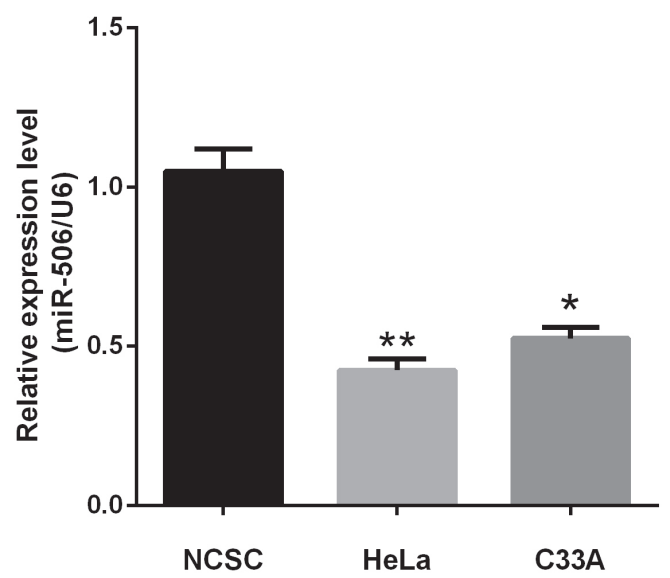

D

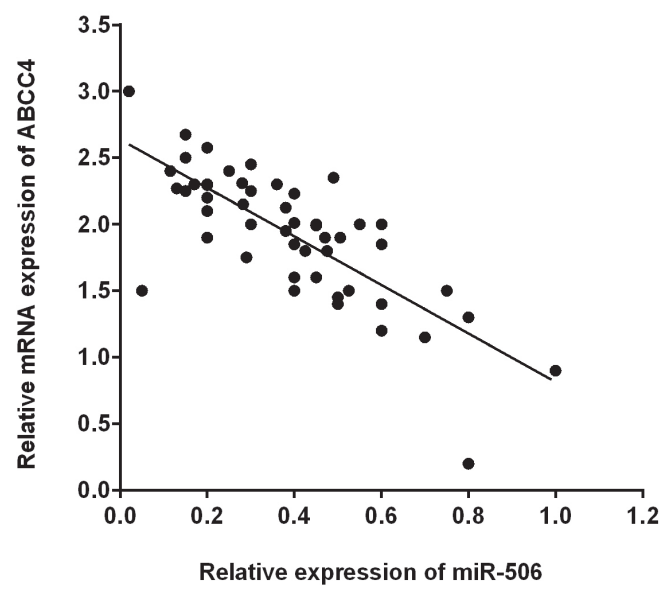

Figure 1. miR-506 expression was down-regulated in cervical cancer tissues and cell lines and inversely correlated with ABCC4. (A) Expression of miR506 in 50 pairs of cervical cancer samples and their adjacent normal tissues. The expression level of miR-506 was detected by qRT-PCR using U6 as the internal control. (B) Expression levels of miR-506 in two cancer cell lines and primary normal cervical squamous cells. (C) Relative mRNA expression of ABCC4 in cervical cancer tissues. (D) Spearman correlation analysis of miR-506 expression and ABCC4 in 50 cervical cancer tissues. ${ }^{\star * *}$ p $<0.001$, ${ }^{* *} \mathrm{p}<0.01,{ }^{*} \mathrm{p}<0.05$

than that in the adjacent normal tissues $(\mathrm{p}<0.001)$. Then, we found that miR-506 expression was inversely correlated with the ABCC4 level in these clinical specimens (Figure 1D). These results indicate that miR-506 is downregulated in CC tissues and that reduced expression of miR-506 and increased ABCC4 level may play an essential part in development and progression of CC. Additionally, miR-506 may have a critical role in regulating $\mathrm{CC}$ cell proliferation. However, the particular function of miR-506 CC is still unknown.

miR-506 inhibits cervical cancer cell proliferation and xenograft tumor growth. As the downregulation of miR-506 is inversely correlated with ABCC4, we hypothesized that miR-506 is a tumor suppressor in CC, and affects CC cells proliferation. HeLa and C33A cells lines were selected and transiently transfected with miR-506 mimic/inhibitor or $\mathrm{miR}-\mathrm{NC}$ and then the expression levels in cells were deter- mined by qRT-PCR. The results showed that the intracellular level of miR-506 was higher in HeLa and C33A cells lines transfected with the miR-506 mimic as compared with the level in cells transfected with miR-NC (Figure $2 \mathrm{~A}, \mathrm{p}<0.01$ ); and in contrast, the expression level of miR-506 was statistically lower in HeLa and C33A cells lines transfected with the miR-506 inhibitor compared with the levels in cells transfected with miR-NC (Figure 2B, $\mathrm{p}<0.01$ ). Meanwhile, the MTT proliferation assay showed that the rate of cell proliferation in the miR-506 mimic transfected group was significantly decreased in comparison with the miR-NC groups (Figure 2C, $\mathrm{p}<0.01$ ). However, there was no difference in the proliferation rate between the cells ( $\mathrm{HeLa}$ and $\mathrm{C} 33 \mathrm{~A}$ ) transfected with miR-506-inhibitor and the cells transfected with miR-NC, although a slight increase was observed in the front two cells (Figure 2D). 
A

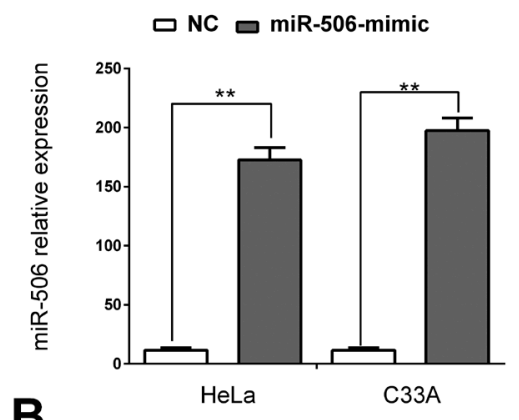

B

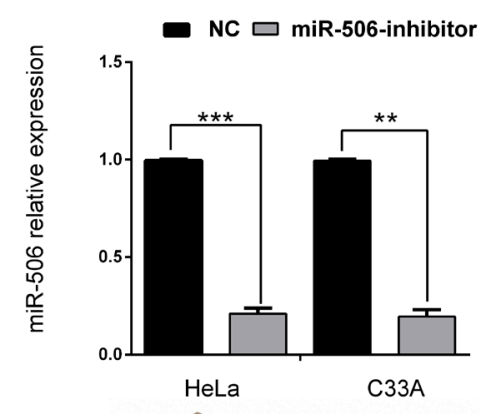

E

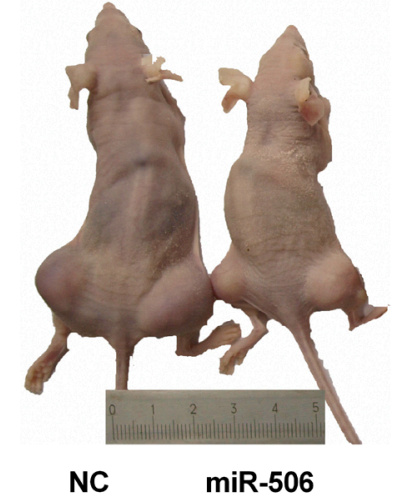

C
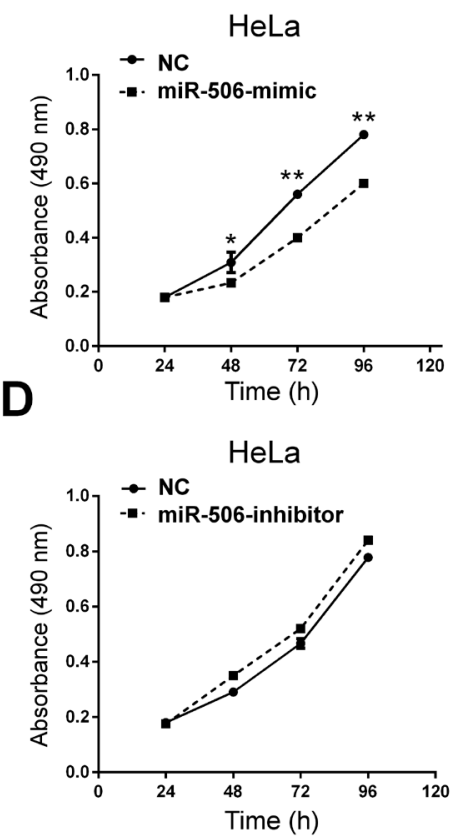

F

NC

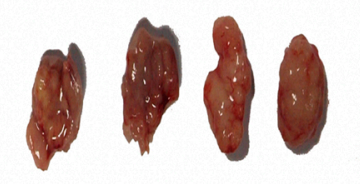

miR-506

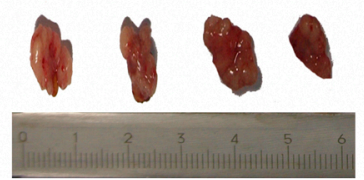

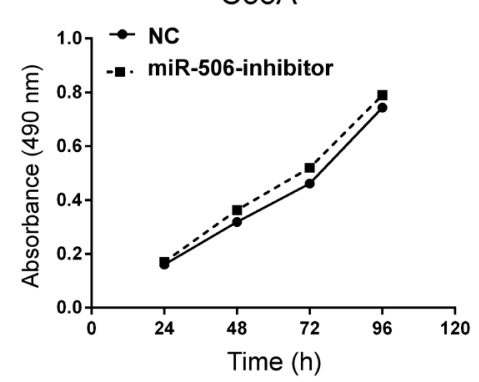

C33A

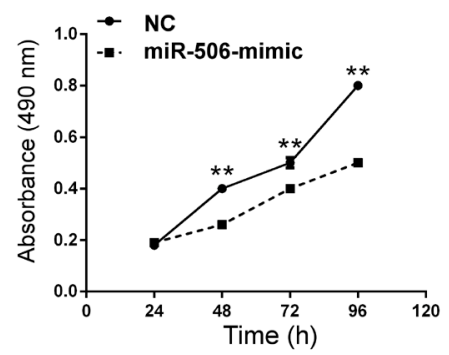

C33A

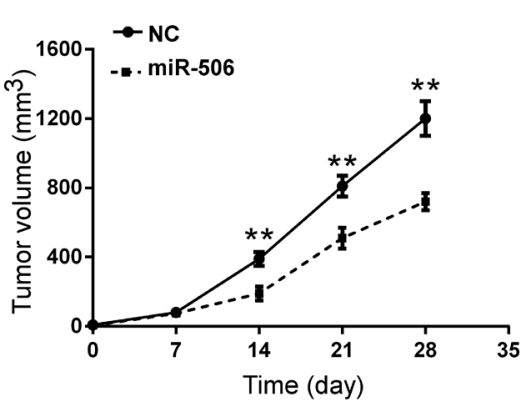

Figure 2. The re-expression of miR-506 induced growth inhibition in cervical cancer cells in vitro. (A-B) miR-506 was re-expressed in HeLa and C33A cell lines and miR-506 levels were determined by qRT-PCR after transfection with miR-506 mimic/inhibitor and negative control (NC). (C-D) MTT assay results showed that miR-506 suppressed viability of HeLa and C33A cells after transfection with miR-506 mimic/inhibitor or miR-ctrl. Mean \pm SD of three independent experiments are shown. (E) Tumors formed in nude mice. HeLa cells stably over-expressing miR-506 or empty vector were injected into the flanks of nude mice $(n=10)$, and the mice were sacrificed after 4 weeks. (F) The volume of the tumors from the mice injected with miR-506-overexpressing HeLa cells was significantly smaller than that in those injected with miR-ctrl-expressing cells. The data are shown as the means \pm S.D. ${ }^{* * *} \mathrm{p}<0.001,{ }^{* *} \mathrm{p}<0.01$

To further monitor the effect of miR-506 over-expression on the CC cell proliferation in vivo, we injected the HeLa cells transfected with miR-506 or miR-control into the dorsal flanks of mice. After 4 weeks, all mice were killed, and the volume of the resulting tumors was measured. The sizes of tumors in HeLa/miR-506 mimic group were significantly smaller than those in the NC groups (Figure 2E, 2F). These observations demonstrated that miR-506 over-expression inhibits cervical cancer cells proliferation both in vitro and in vivo.
miR-506 directly targets ABCC4 by binding to its 3'-UTR. To further expound the potential mechanism of how miR-506 induced the CC cell proliferation, we utilized microRNA.org online and microRNA Targetscan software to predict the targets of miR-506, and then ABCC4 was focused (Figure 3A). Luciferase reporter assay was used to identify ABCC4 as a direct target of miR-506, cells were co-transfected with wild type (WT) pmirGLO-UTR of ABCC4 3'-UTR or mutant type (MT) pmirGLO-UTR of ABCC4 3'-UTR vector and miR-506 mimic/inhibitor, or negative controls (NC). 
The results showed that approximately $22 \%$ and $25 \%$ reduction in luciferase activity was observed in miR-506 mimics transfected HeLa and C33A cells, respectively, whereas the repressive effect of miR-506-inhibitor increased luciferase activity in wild-type ABCC4 by approximately $32 \%$ and $28 \%$, respectively, as compared to the control groups. Meanwhile, the luciferase activities of cells co-transfected with miR-506 (mimics, inhibitor, or NC) and ABCC4 3'-UTR-mut vector were unaffected (Figure 3B). Furthermore, the expression of ABCC4 was decreased in HeLa cells transfected with miR-506 mimics as compared to those with NC-mimic, while the expression was observed to be increased in HeLa cells with miR-506 inhibitor compared to the NC group (Figure 3C). Taken together, these data indicate that ABCC4 acts as a direct target of miR-506.

\section{Discussion}

CC was once considered to be one of the most serious cancers in women worldwide, and almost $90 \%$ of CC deaths occurred in developing scale of the world [2]. Although cancer treatments have been improved in recent years, the outcomes of patients with CC remain unsatisfactory [31]. Thus, identifying new targets for the development of effective therapeutics for CC is urgent. Recently, increasing evidence indicates that miRNAs play vital roles as diagnostics biomarkers and therapeutic targets in human cancers [32], and plenty of miRNAs are dysregulated in cervical cancer [11-13]. In the present study, we found that miR-506 was significantly down-regulated in human CC cell lines (HeLa and C33A) and clinical tumor specimens as compared to the matched

\section{A 5'-UAaAugccgucuaucagguUUUgugcCUUAagaga-3' ABCC4 3'-UTR 5'-UAAAUGCCGUCUAUCAGGUUUUGACAAUUUAAGAGA-3' ABCC4 3'-UTR MT}

B

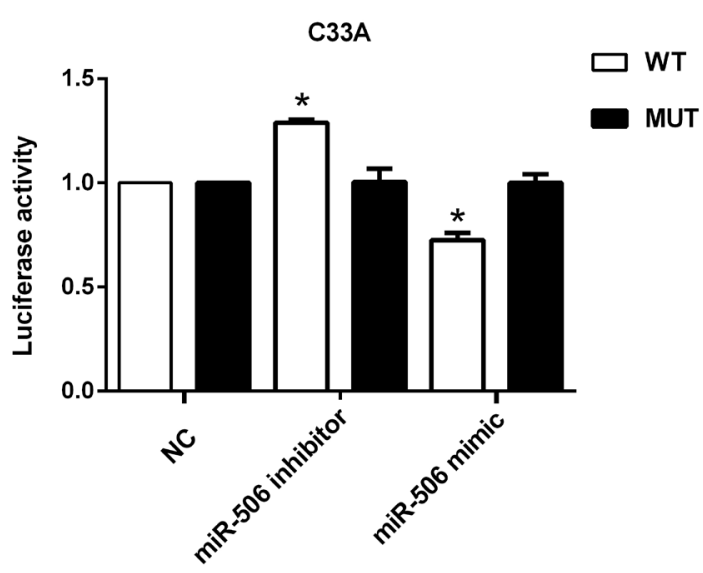

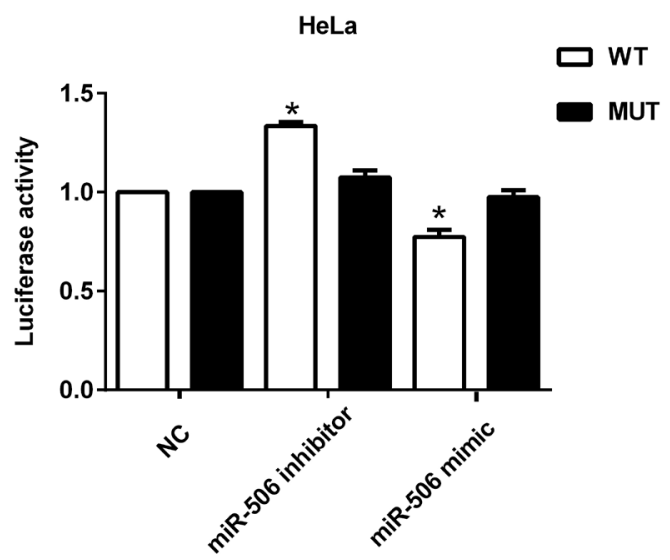

C

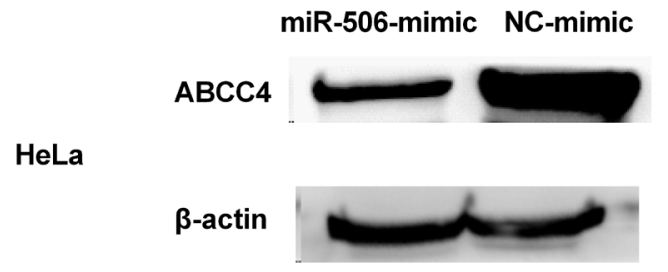

NC-inhibitor miR-506-inhibitor

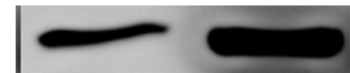

Figure 3. MiR-506 suppressed ABCC4 expression by directly targeting the ABCC4 3'-UTR (A) The binding sites of miR-506 on ABCC4 3'-UTR are predicted by TargetScan. (B) Luciferase reporter assay of HeLa and C33A cells with the pGL3-ABCC4-3'-UTR-wt or pGL3-ABCC4-3'-UTR-mut were co-transfected with miR-506 mimics or NC/inhibitor. ${ }^{\star} p<0.05$ (C) ABCC4 protein expression in HeLa cells transfected with miR-506 mimic/inhibitor or negative control was detected by western blotting analysis. $\beta$-actin served as the loading control. 
cell lines and adjacent normal tissues. We also revealed that up-regulated expression of miR-506 could inhibit CC cell proliferation both in vitro and in vivo. Moreover, ABCC4 was identified as a direct target of miR-506 and the inverse relationship between them was also observed. We demonstrated that miR-506/ABBC4 might act as a novel potential therapeutic target of treatment for $\mathrm{CC}$, and low expression of miR-506 contributes to the tumor progression and tumor cell proliferation in CC patients.

Aberrant expression of miR-506 was reported to be associated with a variety of tumors, including ovarian cancer [33], esophageal cancer [34], colon cancer [35, 36], nasopharyngeal carcinoma [16]. Moreover, Zhang et al have reported that miR-506 was down-regulated and inhibited cell proliferation in $\mathrm{CC}$, but the underlying molecular mechanisms of miR-506 in CC are still elusive. Moreover, a contradictory role of miR-506 has been found. miR-506 acts as an oncogene in melanomas [15], but functions as a tumor suppressor in ovarian cancer [37] In our study, we found that miR-506 expression was down-regulated in CC tissues and cell lines (HeLa and C33A) and inversely correlated with ABCC4 expression by qRT-PCR, suggesting that miR-506 may negatively regulate $\mathrm{CC}$ cell growth.

Wen et al. recently reported that miR-506 could inhibit CC growth, induce cell cycle arrest at the G1/S transition, and enhance apoptosis and chemosensitivity of CC cell lines by targeting Gli3 [21]. Further study in our research found similar results that miR-506 could suppress human CC cells proliferation in vitro and in vivo using MTT assay and animal study, indicating that miR-506 can be a tumor suppressor inhibiting CC proliferation. The data uncovered that the proliferation activity was significantly suppressed in the two cell lines (HeLa and C33A) transfected with miR-506-mimic as compared to the NC group. In cell lines with miR-506inhibitor, however, the proliferation activity just slightly increased without statistical significance when compared to the NC group. The possible reason is that the expression level of miR-506 in CC cell lines used in our study is already very low, the introduction of miR-506-inhibitor would neither down-regulate miR-506 expression nor increase the proliferation activity. But from another aspect, this result featured the down-regulated expression of miR-506 in CC is very common and the degree of suppression is in a large-scale.

miRNAs frequently perform their functions by downregulating the expression of target mRNAs; therefore, we intended to find miR-506 target genes in cervical cancer using microRNA.org online and microRNA Targetscan software. Then, ABCC4 was selected from the two sources. As we know, ABCC4 is an efflux membrane transporter existing in renal tubules, hepatocytes, prostate, blood cells and brain capillaries. Its expression and function are highly variable and are associated with the onset and prognosis of several diseases [38]. Deanna L. Kroetz et al reported that ABCC4 was directly regulated by miR-506 in human kidney [24]. In the present study, using a luciferase reporter system, we observed that miR-506 caused a decrease in the luciferase activity of a wild-type ABCC4 3'UTR reporter, but hardly affected the luciferase activity of mutant ABCC4 3'UTR reporter. Combined with the results of the western blot in this study, these data indicate that miR-506 directly regulates ABCC4 gene expression via binding to the 3'UTR of mRNA, suggesting that the interaction between miR-506 and ABCC4 may have a fundamental biological role.

In conclusion, we have demonstrated that miR-506 acts as a tumor suppressor in CC by reducing cancer growth. Furthermore, we spotted that miR-506 has an inverse correlation with $\mathrm{ABCC} 4$ and directly targets it by binding to its 3'-UTR. This newly identified miR-506 may provide further insight into the progression of $\mathrm{CC}$ and offers a promising therapeutic target for the treatment of CC. A further study to investigate the function of miR-506/ABCC4 axis in tumorigenesis and progression of CC is needed.

\section{References}

[1] BENARD VB, THOMAS CC, KING J, MASSETTI GM, DORIA-ROSE VP et al. Vital signs: cervical cancer incidence, mortality, and screening - United States, 2007-2012. MMWR Morb Mortal Wkly Rep 2014; 63: 1004-1009.

[2] TORRE LA, BRAY F, SIEGEL RL, FERLAY J, LORTETTIEULENT J et al. Global cancer statistics, 2012. CA Cancer J Clin 2015; 65: 87-108. https://doi.org/10.3322/caac.21262

[3] COLOMBO N, CARINELLI S, COLOMBO A, MARINI C, ROLLO D et al. Cervical cancer: ESMO Clinical Practice Guidelines for diagnosis, treatment and follow-up. Ann Oncol 2012; 23 Suppl 7: vii27-32. https://doi.org/10.1093/annonc/mds 268

[4] HILDESHEIM A, WANG SS. Host and viral genetics and risk of cervical cancer: a review. Virus Res 2002; 89: 229-240.

[5] SERRANO-OLVERA A, CETINA L, CORONEL J, DUENAS-GONZALEZ A. Emerging drugs for the treatment of cervical cancer. Expert Opin Emerg Drugs 2015; 20: 165182. https://doi.org/10.1517/14728214.2015.1002768

[6] OJESINA AI, LICHTENSTEIN L, FREEMAN SS, PEDAMALLU CS, IMAZ-ROSSHANDLER I et al. Landscape of genomic alterations in cervical carcinomas. Nature 2014; 506: 371-375. https://doi.org/10.1038/nature12881

[7] CALIN GA, CROCE CM. MicroRNA signatures in human cancers. Nat Rev Cancer 2006; 6: 857-866. https://doi. org/10.1038/nrc1997

[8] SAUMET A, LECELLIER CH. microRNAs and Personalized Medicine: Evaluating Their Potential as Cancer Biomarkers. Adv Exp Med Biol 2015; 888: 5-15. https://doi. org/10.1007/978-3-319-22671-2_2

[9] SRIVASTAVA SK, ARORA S, AVERETT C, SINGH S, SINGH AP. Modulation of microRNAs by phytochemicals in cancer: underlying mechanisms and translational significance. Biomed Res Int 2015; 2015: 848710. https://doi. org/10.1155/2015/848710

[10] VIRANT-KLUN I, STAHLBERG A, KUBISTA M, SKUTELLA T. MicroRNAs: From Female Fertility, Germ Cells, and Stem Cells to Cancer in Humans. Stem Cells Int 2016; 2016: 3984937. https://doi.org/10.1155/2016/3984937 
[11] GALAMB A, BENCZIKM,ZINNERB, VIGHE, BAGHY Ket al. Dysregulation of microRNA expression in human cervical preneoplastic and neoplastic lesions. Pathol Oncol Res 2015; 21: 503-508. https://doi.org/10.1007/s12253-014-9871-x

[12] PEDROZA-TORRES A, LOPEZ-URRUTIA E, GARCIACASTILLO V, JACOBO-HERRERA N, HERRERA LA et al. MicroRNAs in cervical cancer: evidences for a miRNA profile deregulated by HPV and its impact on radio-resistance. Molecules 2014; 19: 6263-6281. https://doi.org/10.3390/ molecules 19056263

[13] GOMEZ-GOMEZ Y, ORGANISTA-NAVA J, GARIGLIO P. Deregulation of the miRNAs expression in cervical cancer: human papillomavirus implications. Biomed Res Int 2013; 2013: 407052. https://doi.org/10.1155/2013/407052

[14] LI J, JU J, NI B, WANG H. The emerging role of miR-506 in cancer. Oncotarget 2016; 7: 62778-62788. https://doi. org/10.18632/oncotarget.11294

[15] STREICHER KL, ZHU W, LEHMANN KP, GEORGANTAS RW, MOREHOUSE CA et al. A novel oncogenic role for the miRNA-506-514 cluster in initiating melanocyte transformation and promoting melanoma growth. Oncogene 2012; 31: 1558-1570. https://doi.org/10.1038/onc.2011.345

[16] ZHANG Z, MA J, LUAN G, KANG L, SU Y et al. MiR-506 suppresses tumor proliferation and invasion by targeting FOXQ1 in nasopharyngeal carcinoma. PLoS One 2015; 10: e0122851. https://doi.org/10.1371/journal.pone.0122851

[17] SUNY,MEZZANZANICAD,ZHANGW.MiR-506: A Multitasker in Suppression of the Epithelial-to-Mesenchymal Transition. RNA Dis 2014; 1: e447. https://doi.org/10.14800/rd.447

[18] CASTRO RE, RODRIGUES CM. Targeting miR-506 in primary biliary cirrhosis to support the HCO3- umbrella. Clin Res Hepatol Gastroenterol 2012; 36: 402-404. https://doi. org/10.1016/j.clinre.2012.05.019

[19] ARORA H, QURESHI R, PARK WY. miR-506 regulates epithelial mesenchymal transition in breast cancer cell lines. PLoS One 2013; 8: e64273. https://doi.org/10.1371/journal. pone.0064273

[20] SAKIMURA S, SUGIMACHI K, KURASHIGE J, UEDA M, HIRATA $\mathrm{H}$ et al. The miR-506-Induced Epithelial-Mesenchymal Transition is Involved in Poor Prognosis for Patients with Gastric Cancer. Ann Surg Oncol 2015; 22 Suppl 3: S1436-1443. https://doi.org/10.1245/s10434-015-4418-2

[21] WENSY,LINY,YUYQ,CAOSJ,ZHANG Retal.miR-506acts as a tumor suppressor by directly targeting the hedgehog pathway transcription factor $\mathrm{Gli} 3$ in human cervical cancer. Oncogene 2015; 34: 717-725. https://doi.org/10.1038/onc.2014.9

[22] RIUS M, THON WF, KEPPLER D, NIES AT. Prostanoid transport by multidrug resistance protein 4 (MRP4/ ABCC4) localized in tissues of the human urogenital tract. J Urol 2005; 174: 2409-2414. https://doi.org/10.1097/01. ju.0000180411.03808.cb

[23] BAI J, LAI L, YEO HC, GOH BC, TAN TM. Multidrug resistance protein 4 (MRP4/ABCC4) mediates efflux of bimaneglutathione. Int J Biochem Cell Biol 2004; 36: 247-257.

[24] MARKOVA SM, KROETZ DL. ABCC4 is regulated by microRNA-124a and microRNA-506. Biochem Pharmacol 2014; 87: 515-522. https://doi.org/10.1016/j.bcp.2013.10.017
[25] SHAFAGHAT F, ABBASI-KENARSARI H, MAJIDI J, MOVASSAGHPOUR AA, SHANEHBANDI D et al. Preparation of Proper Immunogen by Cloning and Stable Expression of cDNA coding for Human Hematopoietic Stem Cell Marker CD34 in NIH-3T3 Mouse Fibroblast Cell Line. Adv Pharm Bull 2015; 5: 69-75. https://doi.org/10.5681/apb.2015.009

[26] LIVAK KJ, SCHMITTGEN TD. Analysis of relative gene expression data using real-time quantitative PCR and the 2(-Delta Delta C(T)) Method. Methods 2001; 25: 402-408. https://doi.org/10.1006/meth.2001.1262

[27] SURDZIEL E, EDER M, SCHERR M. Lentivirus-mediated antagomir expression. Methods Mol Biol 2010; 667: 237248. https://doi.org/10.1007/978-1-60761-811-9_16

[28] ABBAS-TERKI T, BLANCO-BOSE W, DEGLON N, PRALONG W, AEBISCHER P. Lentiviral-mediated RNA interference. Hum Gene Ther 2002; 13: 2197-2201. https:// doi.org/10.1089/104303402320987888

[29] CHEN J, CHEN H, ZHAN Y, YANG X, YU M. [Lentivirusmediated interference of E3 ubiquitin ligase RNF31 inhibits tumor-necrosis factor-alpha-induced activation of nuclear factor-kappaB pathway]. Nan Fang Yi Ke Da Xue Xue Bao 2014; 34: 1713-1720.

[30] KUTNER RH, ZHANG XY, REISER J. Production, concentration and titration of pseudotyped HIV-1-based lentiviral vectors. Nat Protoc 2009; 4: 495-505. https://doi. org/10.1038/nprot.2009.22

[31] ROB L, HALASKA M, ROBOVA H. Nerve-sparing and individually tailored surgery for cervical cancer. Lancet Oncol 2010; 11: 292-301. https://doi.org/10.1016/S14702045(09)70191-3

[32] GILABERT-ESTELLES J, BRAZA-BOILS A, RAMON LA, ZORIO E, MEDINA $\mathrm{P}$ et al. Role of microRNAs in gynecological pathology. Curr Med Chem 2012; 19: 2406-2413.

[33] LIU G, SUN Y, JI P, LI X, COGDELL D et al. MiR-506 suppresses proliferation and induces senescence by directly targeting the CDK4/6-FOXM1 axis in ovarian cancer. J Pathol 2014; 233: 308-318. https://doi.org/10.1002/path.4348

[34] YAO WJ, WANG YL, LU JG, GUO L, QI B et al. MicroRNA-506 inhibits esophageal cancer cell proliferation via targeting CREB1. Int J Clin Exp Pathol 2015; 8: 10868-10874.

[35] ZHANG Y, LIN C, LIAO G, LIU S, DING J et al. MicroRNA-506 suppresses tumor proliferation and metastasis in colon cancer by directly targeting the oncogene EZH2. Oncotarget 2015; 6: 32586-32601. https://doi.org/10.18632/oncotarget.5309

[36] TONG JL, ZHANG CP, NIE F, XU XT, ZHU MM et al. MicroRNA 506 regulates expression of PPAR alpha in hydroxycamptothecin-resistant human colon cancer cells. FEBS Lett 2011; 585: 3560-3568. https://doi.org/10.1016/j. febslet.2011.10.021

[37] YANG D, SUN Y, HU L, ZHENG H, JI P et al. Integrated analyses identify a master microRNA regulatory network for the mesenchymal subtype in serous ovarian cancer. Cancer Cell 2013; 23: 186-199. https://doi.org/10.1016/j. ccr.2012.12.020

[38] KURZAWSKI M, DZIEDZIEJKO V, POST M, WOJCICKI $\mathrm{M}$, URASINSKA E et al. Expression of genes involved in xenobiotic metabolism and transport in end-stage liver disease: up-regulation of ABCC4 and CYP1B1. Pharmacol Rep 2012; 64: 927-939. 